\title{
Suppression of relaxin receptor RXFP1 decreases prostate cancer growth and metastasis
}

\author{
Shu Feng ${ }^{1,2}$, Irina $U$ Agoulnik ${ }^{3,10}$, Anne Truong ${ }^{1}$, Zhen $L^{1}{ }^{1}$, \\ Chad J Creighton ${ }^{4}$, Elena M Kaftanovskaya ${ }^{10}$, Rhea Pereira ${ }^{10}$, \\ Hee Dong Han ${ }^{5}$, Gabriel Lopez-Berestein ${ }^{6,7}$, Thomas Klonisch', \\ Michael M Ittmann ${ }^{2,9}$, Anil K Sood ${ }^{5,7}$ and Alexander I Agoulnik ${ }^{1,10}$
}

\footnotetext{
${ }^{1}$ Department of Obstetrics and Gynecology, ${ }^{2}$ Department of Pathology and Immunology and ${ }^{3}$ Department of Molecular and Cellular Biology, Baylor College of Medicine, Houston, Texas 77030, USA

${ }^{4}$ Baylor College of Medicine, Dan Duncan Cancer Center, Houston, Texas 77030, USA

${ }^{5}$ Departments of Gynecologic Oncology and Cancer Biology, ${ }^{6}$ Department of Experimental Therapeutics and ${ }^{7}$ Center for RNA Interference and Non-Coding RNA, MD Anderson Cancer Center, University of Texas, Houston, Texas 77030, USA

${ }^{8}$ Department of Human Anatomy and Cell Science, University of Manitoba, Winnipeg, Manitoba R3E 0J9, Canada

${ }^{9}$ Michael E DeBakey Veterans Affairs Medical Center, Houston, Texas 77030, USA

${ }^{10}$ Department of Human and Molecular Genetics, Herbert Wertheim College of Medicine, Florida International University, Miami, Florida 33199, USA
}

(Correspondence should be addressed to A I Agoulnik at Department of Human and Molecular Genetics, Herbert Wertheim College of Medicine, Florida International University; Email: aagoulni@ fiu.edu)

\begin{abstract}
Relaxin (RLN) is a small peptide hormone expressed in several cancers of reproductive and endocrine organs. Increased expression of RLN in prostate cancer correlates with aggressive cancer. RLN G-protein-coupled receptor (RLN family peptide receptor 1, RXFP1) is expressed in both androgen receptor (AR)-positive and -negative prostate cancers as well as in prostate cancer cell lines. RLN behaves as a cell growth factor and increases invasiveness and proliferation of cancer cells in vitro and in vivo. The objective of this study is to determine whether downregulation of RXFP1 expression using small interfering RNA (siRNA) reduces cancer growth and metastasis in a xenograft model of prostate cancer. We used two well-characterized prostate adenocarcinoma cell lines, AR-positive LNCaP cells and AR-negative PC3 cells. The tumors were established in nude male mice by s.c. injections. Intratumoral injections of siRNAs loaded on biodegradable chitosan nanoparticles led to a downregulation of RXFP1 receptor expression and a dramatic reduction in tumor growth. In LNCaP tumors, the siRNA treatment led to an extensive necrosis. In PC3 xenografts treated with siRNA against RXFP1, the smaller tumor size was associated with the decreased cell proliferation and increased apoptosis. The downregulation of RXFP1 resulted in significant decrease in metastasis rate in PC3 tumors. Global transcriptional profiling of PC3 cells treated with RXFP1 siRNA revealed genes with significantly altered expression profiles previously shown to promote tumorigenesis, including the downregulation of MCAM, MUC1, ANGPTL4, GPI, and TSPAN8. Thus, the suppression of RLN/RXFP1 may have potential therapeutic benefits in prostate cancer.
\end{abstract}

Endocrine-Related Cancer (2010) 17 1021-1033

\section{Introduction}

Prostate cancer is the most common type of cancer in men in the United States and the second leading cause of cancer deaths. Androgens play a key role in prostate cancer initiation and progression and androgen ablation is initially effective but once advanced to the androgen-independent stage prostate cancer becomes nonresponsive to androgen ablation therapy. The identification of novel endogenous factors involved in the proliferation, viability, and migration of prostate cancer cells may create potential therapeutic targets for future treatment. The prostate is one of the main sites 
of relaxin (RLN) expression in males (Agoulnik 2007). Originally, this peptide hormone was identified by its effects on the relaxation (hence the name) of pubic symphysis, cervical softening, and the inhibition of spontaneous myometrial contractions during pregnancy and parturition in females (Sherwood 2004). New roles for RLN in various other physiological scenarios have recently been established. These include regulation of connective tissue remodeling and fibrosis, promotion of angiogenesis, blood vessels dilation, chronotropic action in the heart, wound healing, inhibition of apoptosis, and other physiological effects (Sherwood 2004, Bathgate et al. 2006). There are two almost identical RLN genes in the genome of great apes and humans (RLN1 and RLN2); all other studied mammalian species have only one copy of the orthologous gene (Klonisch et al. 2001). RLN2 is the only circulating form of RLN detected in peripheral blood. Both the RLN genes are expressed in the prostate; however, the RLN1 expression is significantly lower (Hansell et al. 1991). Although sharing only $48 \%$ identity at the amino acid level, mouse RLN1 and human RLN2 are each fully bioactive in both species (Feng et al. 2005). RLN family peptide receptor 1 (RXFP1) is a G-proteincoupled receptor expressed on the cell surface (Hsu et al. 2002). Stimulation of RXFP1 with RLN causes a cAMP increase mediated through $G_{s} / G_{i}$ proteins and protein kinase A in some cells (Bathgate et al. 2006). In addition, the involvement of tyrosine kinases, protein kinase $\mathrm{C}$ zeta, phosphatidylinositol 3-kinase (PI3K), and other molecules in RLN signaling was also demonstrated (Bathgate et al. 2006). RLN regulates a number of critical molecules directly relevant to tumor progression. These molecular targets of RLN signaling include collagens and various metalloproteinases (MMPs), tissue inhibitors of MMPs, vascular endothelial growth factor, insulinlike growth factor-binding protein 1, hyaluronic acid, prolactin, laminin, nitric oxide synthase, S100A4, and others (Klonisch et al. 2007).

An increased expression of RLN has been reported in breast, endometrial, thyroid, and prostate cancers especially in patients with aggressive metastatic disease (Klonisch et al. 2007). The overall survival was shorter in patients with increased expression of RLN (Kamat et al. 2006, Feng et al. 2007). The in vitro invasiveness of carcinoma cells was significantly increased on incubation with RLN or in cells transfected with RLN expression constructs (Kamat et al. 2006, Silvertown et al. 2006, Feng et al. 2007). The stimulating effect on growth and angiogenesis of lentiviral-delivered RLN in the prostate carcinoma cell line PC3 was recently demonstrated in vivo (Silvertown et al. 2006). Furthermore, we have demonstrated that the survival of transgenic adenocarcinoma of mouse prostate (TRAMP) mice with transgenic RLN overexpression was decreased (Feng et al. 2007). Neuroendocrine differentiation of prostate adenocarcinoma $\mathrm{LNCaP}$ cells and the presence of $\mathrm{p} 53$ gain-of-function mutant allele R273H in LNCaP cells coincide with an increase in RLN expression (Vinall et al. 2006). It was suggested that RLN might be negatively regulated by androgens in vitro and in vivo. Furthermore, PI3K/Akt signaling and components of the Wnt pathway can facilitate RLN-mediated activation of the androgen receptor (AR) pathway (Liu et al. 2008). On the other hand, small interfering RNA (siRNA)-mediated downregulation of endogenous RXFP1 expression in PC3 and LNCaP cells decreased cell invasion and proliferation and increased cell apoptosis in vitro (Feng et al. 2007).

Based on the available data, we hypothesized that the suppression of RLN signaling in prostate cancer cells might affect tumor growth and metastasis in vivo. In the present study, we analyzed the effect of RLN receptor RXFP1 downregulation on prostate cancer progression using an in vivo model. Tumor growth and metastasis were analyzed in AR-positive LNCaP and AR-negative prostate adenocarcinoma PC3 cells transplanted into nude mice treated with RXFP1specific small interfering RNA (siRNA) (siRXFP1) incorporated into chitosan nanoparticles (CNNP) to inhibit RXFP1 expression. Our data indicate that RXFP1 may be a promising new target in prostate cancer therapy.

\section{Materials and methods}

\section{Cell culture}

LNCaP and PC3 cells were originally purchased from American Type Culture Collection, Inc. (Manassas, VA, USA). LNCaP cells were maintained in RPMI-1640 medium while PC3 cells were cultured in DMEM/F12 medium (Invitrogen) supplemented with $10 \%$ fetal bovine serum and $1 \%$ antibiotics mix (100 U penicillin/ml and $100 \mu \mathrm{g}$ streptomycin/ml; Invitrogen) at $37{ }^{\circ} \mathrm{C}$ in a humidified atmosphere of $95 \%$ air with $5 \% \mathrm{CO}_{2}$.

\section{siRNA transfection in vitro}

In this project, we have used two siRNAs targeting RXFP1 expression. The siRXFP1-0 GGAUGUCAAGUGCUCCCUUtt (sense strand), a highly efficient siRNA was identified previously (Kamat et al. 2006, 
Feng et al. 2007). Another one was selected after testing four HP GenomeWide siRNAs (Qiagen) targeting human RXFP1 (Hs_LGR7_1,2,3, and 5 HP siRNA) in LNCaP cells. The siRXFP1-5 siRNA, ACGUGACACGUUCGGAGAAtt, was selected for this project (Supplementary Figure 1, see section on supplementary data given at the end of this article). The siRXFP1-0 and siRXFP1-5 were synthesized by Sigma-Genosys and Qiagen respectively. Negative control siRNA (siNC) with no significant sequence similarity to human gene sequences was used as control (Qiagen). LNCaP or PC3 cells were seeded on $100 \mathrm{~mm}$ dishes $24 \mathrm{~h}$ before transfection. siRXFP1 and siNC (5 $\mu \mathrm{g}$ each) were transfected into the cells using the Cell Line Nucleofector kit V (PC3) or kit $\mathrm{R}$ (LNCaP) with the nucleofector device (Lonza Group Ltd, Basel, Switzerland) according to the manufacturer's instruction. Transfected cells were harvested after $48 \mathrm{~h}$, and total RNA was isolated for cDNA microarray assay and quantitative real-time reverse transcription-PCR (qRT-PCR).

\section{Incorporation of SiRNA into chitosan nanoparticles}

CNNP are formed spontaneously upon the addition of aqueous tripolyphosphate (TPP) solution to chitosan solution under magnetic stirring at $200 \mathrm{~g}$ and mixed for a further $10 \mathrm{~min}$ after addition of TPP. siRNA was added to the TPP solution in a 3:1 w:w ratio under constant magnetic stirring ( $200 \mathrm{~g}$ ) at room temperature. The chitosan preparation was then centrifuged at $9000 \mathrm{~g}$ for $30 \mathrm{~min}$ at $5{ }^{\circ} \mathrm{C}$. Supernatants were discarded and the pellet containing the CNNP was washed three times with distilled water to remove any residual sodium hydroxide, and finally resuspended in ultrapure water. The preparations consisted of control siRNA incorporated in CNNP (siNC-CNNP) and siRNA RXFP1 incorporated in CNNP (siRXFP1-CNNP).

\section{Animal experiments}

All experiments were conducted using the standards for human care in accordance with the NIH Guide for the Care and Use of Laboratory Animals and approved by the Baylor College of Medicine Institutional Committee. The xenograft experiments were performed on nude mice obtained from the NCI Animal Production Program. LNCaP or PC3 cells $\left(10^{7}\right)$ (per mouse/injection site) suspended in $200 \mu \mathrm{l}$ of a $1: 1$ mixture of RPMI-1640 or DMEM/F12 and Matrigel (BD Biosciences, San Jose, CA, USA) were injected s.c. into the right flank of nude mice. The tumor size was measured by a digital slide caliper and the tumor volume in cubic millimeters was calculated by the following formula: volume $=$ width $^{2} \times$ length $/ 2$. When tumors became visible at 2 weeks (PC3) and at 4 weeks (LNCaP), the animals were randomized into two groups. For experiments with LNCaP cells, 45 mice were injected and 18 developed tumors. In the xenograft experiments using PC3 cells, a total of 45 mice were used in two consecutive experiments (40 tumors). Tumor-bearing nude mice were treated with siRXFP1-CNNP and siNC-CNNP. For the LNCaP xenografts, we used an equal amount of siRNAs (RXFP1-0 and RXFP1-5), whereas PC3 tumors were treated with siRXFP1-0 only. Four micrograms of siRNA CNNP were diluted in $100 \mu \mathrm{l}$ of $0.9 \%$ saline before injection and a final volume of $100 \mu \mathrm{l}$ was applied directly into the tumor as a bolus injection. Each siRNA species incorporated into the CNNP was injected intratumorally twice a week. Mice with $\mathrm{LNCaP}$ tumors were treated for 21 days (seven injections) and were killed 2 days after the last treatment. Mice with PC3 tumors received a total of six injections and were killed 9 days after the last treatment. All mice were analyzed for the presence of macroscopic and microscopic metastasis in the abdominal organs, the thorax, and the brain. The xenograft tumors, lung, liver, kidneys, brain, and regional lymph nodes were collected and tumor weights were measured.

\section{Histological analysis and immunohistochemistry}

Xenograft tumor tissues were fixed in cold $4 \%$ paraformaldehyde (PFA) and were embedded in paraffin. Prior to the histological analysis, tissue sections $(5 \mu \mathrm{m})$ were deparaffinized in xylene and a descending alcohol series and were stained with hematoxylin and eosin. Staining with picrosirius red was performed to examine the density of collagen in the extracellular matrix (ECM; EMS, Hatfield, PA, USA).

Tumor cell proliferation in xenograft tumors treated with RXFP1 siRNA was evaluated using Ki67 immunodetection. The expression of RXFP1 was evaluated using a monoclonal RXFP1 antibody. Briefly, tissue sections were deparaffinated and antigen retrieval was performed by boiling slides in $10 \mathrm{mmol} / \mathrm{l}$ sodium citrate buffer ( $\mathrm{pH}$ 6.0) for $20 \mathrm{~min}$. Nonspecific binding sites were blocked with 5\% BSA in PBS for $1 \mathrm{~h}$ at room temperature. Slides were incubated with anti-rabbit Ki67 (1:200; Abcam, Cambridge, MA, USA) or RXFP1/LGR7 monoclonal antibody M01, clone 3E3 (1:200; Abnova, Taipei, Taiwan) for $1 \mathrm{~h}$ at room temperature and incubated for $45 \mathrm{~min}$ 
with an appropriate HRP-conjugate/HRP-substrate stain (Pierce, Rockford, IL, USA). Slides were counterstained with hematoxylin and images were taken with a Nikon-TMS inverted microscope equipped with Olympus DP70 digital imaging system. Ki67 staining was scored by the percentage of positive tumor cells.

Terminal deoxynucleotidyl transferase-mediated dUTP nick end labeling assay was used for the detection of apoptosis in PC3 xenograft tumors using ApopTag In Situ Apoptosis Detection kit (Serologicals Corp., Norcross, GA, USA). The mouse anti-human cluster of differentiation 34 (CD34) monoclonal antibody (1:300; Dako North America, Carpinteria, CA, USA) was used to identify vascular cells in tumor sections. A vessel was defined as an open lumen lined by one or more CD34-positive cells.

Eight random fields per section were scored at $\times 400$ magnification. Scoring was performed by two independent researchers in a blind manner. In the LNCaP xenograft experiment, seven siRXFP1 and ten siNC tumors were analyzed. In the PC3 xenograft experiment, the analysis was performed with 12/15 (Ki67), 8/8 (apoptosis), and 10/10 (CD34) mice per group, siRXFP1/siNC.

To analyze an efficiency of siRNA gene knockdown in vitro, LNCaP cells were transfected with siRXFP1-0, siRXFP1-5, and siNC (5 $\mu$ g each) using the Amaxa Cell Line Nucleofector kit R (Lonza) according to the manufacturer's manual and were grown in poly-D-lysine-coated six-well plates. After $48 \mathrm{~h}$, cells were washed with PBS and fixed for $10 \mathrm{~min}$ with $4 \%$ paraformaldehyde. The wells were washed twice with PBS and then incubated in $5 \% \mathrm{BSA} / 0.3 \mathrm{M}$ glycine in $0.1 \%$ PBS-Tween for $1 \mathrm{~h}$ to increase cell permeability and block nonspecific protein-protein interactions. The cells were incubated with the RXFP1 antibody $(1: 200)$ overnight at $+4{ }^{\circ} \mathrm{C}$. Detection was performed by a Vectastain $\mathrm{ABC}$ kit (Vector Laboratories Inc, Burlingame, CA, USA). Cells were counterstained with Harris hematoxylin. Wells were mounted for microscopic examination.

\section{RNA isolation and cDNA synthesis}

Total RNA was isolated from PC3 and LNCaP cells transfected with siRNA or from xenografts using the RNeasy kit (Qiagen). For the expression array studies, the PC3 cell RNA was digested with the DNA-free DNase Treatment and Removal Reagents (Ambion, Austin, TX, USA) according to the manufacturer's protocol. cDNA was synthesized using an oligo(dT) primer and RETROscript kit (Ambion).

\section{Real-time quantitative RT-PCR}

Real-time qRT-PCR was performed according to a qPCR SybrGreen real-time protocol on the IQ5 iCycler (Bio-Rad). Primers for different genes are listed in Supplementary Table 1, see section on supplementary data given at the end of this article. The GAPDH expression was used for normalization of SybrGreen data. RXFP1 expression level was estimated using TaqMan Gene Expression Assay Hs00222171 (Applied Biosystems, Foster City, CA, USA) in LNCaP cells or qPCR SybrGreen protocol in PC3 cells as described before (Kamat et al. 2006). For normalization of mouse Rxfpl expression, we used ubiquitously expressed hydroxymethylbilane synthase gene Hmbs (or Pbgd). The relative fold change in mRNA level was calculated by the comparative $C_{\mathrm{t}}\left(2^{-\Delta \Delta \mathrm{C}_{\mathrm{t}}}\right)$ method. Each measurement point was repeated at least in triplicate for three samples and the average and S.E.M were calculated.

\section{Expression microarray analysis}

Gene expression profiles were analyzed using the Illumina HumanRefseq-8 Expression BeadChip platform (Illumina, San Diego, CA, USA). Each profile represented RNA from pooled biological triplicate samples (two profiles per group, six biological replicates in total for each group). Two hundred nanograms of total RNA were amplified and purified using Illumina TotalPrep RNA Amplification kit (Ambion) following manufacturer's instructions. In vitro transcription was performed and biotinylated cRNA was synthesized during $14 \mathrm{~h}$ amplification with dNTP mix containing biotin-dUTP and T7 RNA polymerase. Amplified cRNA was subsequently purified and the concentration was measured with a NanoDrop ND-1000 Spectrophotometer (NanoDrop Technologies, Wilmington, DE, USA). Aliquots of $750 \mathrm{ng}$ of amplified product were loaded onto Illumina Sentrix Beadchip Array Human RefSeq- 8 arrays, hybridized at $58^{\circ} \mathrm{C}$ in an Illumina Hybridization Oven (Illumina) for 16-20 h, washed, and incubated with streptavidin-Cy3 to detect biotin-labeled cRNA on the arrays. Arrays were dried and scanned with BeadArray Reader (Illumina). Data were analyzed using BeadStudio software (Illumina). Expression of selected genes was confirmed by qRT-PCR. Expression data were quantile normalized (using software provided by Giordano et al. (2003) and Saldanha (2004)) and expression patterns were visualized as a heat map using Java TreeView (Saldanha 2004). 


\section{Statistical analysis}

Student's $t$-test and ANOVA were used to assess significance of differences among the different groups. Differences were expressed as mean \pm s.E.M. with $P$ values smaller than 0.05 being considered as statistically significant.

\section{Results}

\section{Selection of siRNA and analysis of RXFP1 suppression in vitro and in vivo}

The expression of RXFP1 RLN receptor in $\mathrm{LNCaP}$ and PC3 cells has been established previously (Feng et al. 2007). We identified one siRNA (siRXFP1-0) capable of downregulating the expression of RXFP1 in a variety of prostate and endometrial cancer cells in vitro (Kamat et al. 2006, Feng et al. 2007). Additional four siRNAs obtained from Qiagen were tested in LNCaP cells (Supplementary Figure 1A). All four constructs significantly downregulated the RXFPl expression $48 \mathrm{~h}$ after transfection as determined by qRT-PCR with RXFP1-specific primers. The GAPDH expression was used for normalization. The immunohistochemistry (IHC) with specific anti-RXFP1 monoclonal antibodies confirmed the significant downregulation of the receptor in cells transfected with siRXFP1 (Supplementary Figure 1B).

We next assessed the efficiency of siRNA RXFPl gene targeting in vivo using siRNAs incorporated

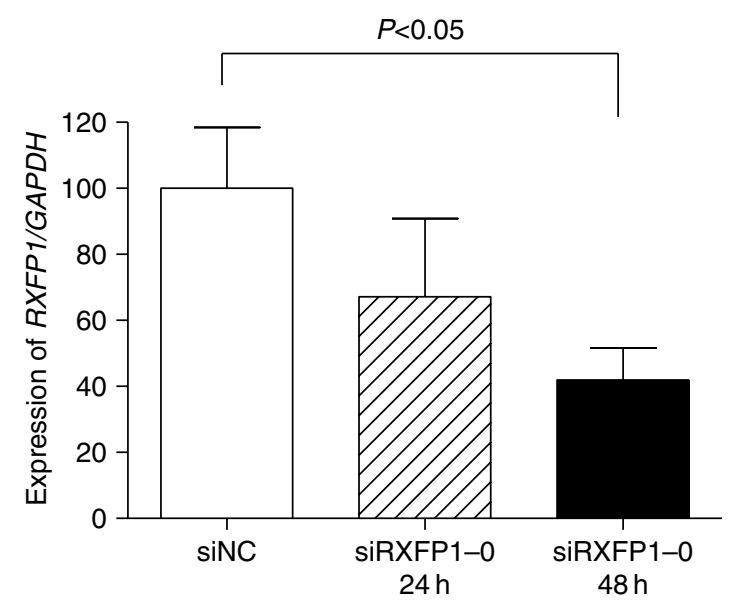

Figure 1 Downregulation of RXFP1 expression by siRNAs in vivo. Intratumoral injection of siRXFP1-0-CNNP inhibited $R X F P 1$ expression in PC3 xenograft tumor. RXFP1 mRNA expression in PC3 xenograft was evaluated by qRT-PCR 24 and $48 \mathrm{~h}$ after siRNA injection ( $n=3$ in each group). Error bars denote \pm S.E.M. into CNNP (siRXFP1-CNNP). We analyzed the effect of the siRXFP1-0-CNNP injection on RXFP1 mRNA expression in tumor cells. PC3 cells $\left(10^{7} /\right.$ injection site $)$ were injected s.c. into the flanks of nine nude male mice. When tumors became clearly visible at 14 day, a single intratumoral injection of siRXFP1-0-CNNP or siNC-CNNP was administered. Analysis of RXFP1 transcript levels in xenograft tumor mRNA extracts was performed by qRT-PCR and clearly showed a downregulation of gene expression in the siRXFP1 group, which reached statistical significance after $48 \mathrm{~h}$ as compared with siNC-treated tumor tissue $(P<0.05$; Fig. 1). Thus, we confirmed our previous data on the dynamic stability and suppressive effects of chitosan siRNA (Lu et al. 2010). Based on these results, we decided to proceed with a bi-weekly injection schedule.

\section{Effect of siRXFP1 on in vivo growth of LNCaP xenografts}

Forty-five male nude mice received s.c. injection of $10^{7}$ LNCaP cells per injection site. After 28 days, 18 mice developed well-defined tumors and were divided into two groups with the same average tumor volume. Tumors were injected either with siNC-CNNP complexes or with a mix of siRXFP1-0 and siRXFP1-5 siRNA-CNNP complexes twice a week (seven injections). As shown in Fig. 2A, the tumors injected with control siNC grew faster and by the end of 23 day reached the criteria for euthanasia. Tumors treated with siRXFP1 increased in volume within 3-7 days after the first injection. However, at the end of 21 day, tumor volumes were slightly lower than at the beginning of experiment. The growth curves of the tumors in this experimental group were significantly different from controls $(P<0.0001)$ and the average tumor volume at the end of experiment was $60 \%$ lower than in the control group. No animal mortality or morbidity was observed for the duration of the experiments suggesting that the treatment with siRNA-CNNP complexes was not toxic to the animals under the applied conditions.

Two days after the last injection, mice were killed and the primary tumors were extracted. Tumor weight of the siRXFP1-treated group was significantly lower than that of the siNC group (64\% reduction, $P=0.029$, Fig. 2B). Examination of the mice at both the macroand microscopic levels did not reveal any metastases in the abdominal organs, the cervical nodes, the thorax, or the brain in control or experimental animals. Histological analysis of the majority of tumors treated with 


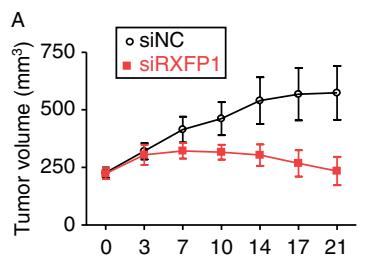

C

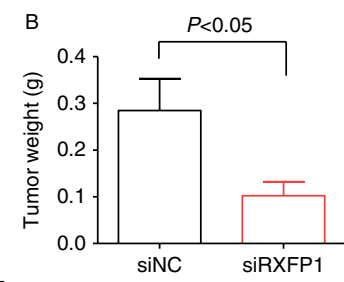

D

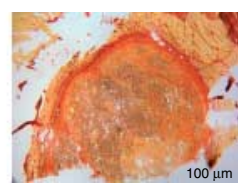

siNC

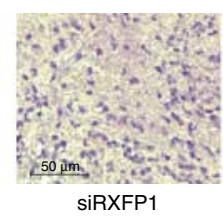

Figure 2 Suppression of in vivo tumor growth by siRXFP1-CNNP in androgen-sensitive LNCaP cells.

(A) LNCaP cells $\left(10^{7}\right.$ cells/injection site) in $200 \mu \mathrm{l}$

RPMI-1640:Matrigel B (1:1) were inoculated s.c. into the right flank of nude mice. Four weeks later, the tumor-borne mice were treated with a mix of SiRXFP1-0 and siRXFP1-5-CNNP $(n=8)$ complexes and control siNC-CNNP $(n=10)$ bi-weekly (seven injections). Tumor volumes were measured by digital caliper. The difference in the tumor volume was significant $(P<0.05)$ from days 14 to 21 . (B) A $64 \%$ reduction in tumor weight was observed in the siRXFP1-treated group after resection at the end of experiment $(P<0.05)$. (C) A reduction in cell density was detected in SiRXFP1-CNNP-treated tumors. Representative sections stained with hematoxylin and eosin are shown. (D) Small tumors in the siRXFP1-CNNP-treated group encapsulated in a thick collagen layer are shown with picrosirius red staining. Magnification, $\times 400$. Error bars denote \pm s.E.M.

siRXFP1-CNNP revealed extensive vacuolated areas with granulation and evidence of necrosis. In contrast, the control siNC-CNNP-treated tumors were mostly dense and composed primarily of sheets of epithelial cells (Fig. 2C). The presence of vacuolated and necrotic areas within the tumors on treatment suggested that the siRNA-CNNP nanoparticles were taken up and likely caused the adverse cellular effects observed in close vicinity of the injection sites. The small tumors were encapsulated in a thick collagenpositive layer (Fig. 2D).

The examination of human $R X F P l$ and mouse $R x f p l$ gene expressions in primary tumors was performed using qRT-PCR. The specificity of the human and mouse primers was confirmed using LNCaP RNA and the RNA isolated from mouse gubernaculum (data not shown). Treatment of the tumors with siRXFP1-CNNP complexes sharply reduced the expression of the human target gene (30\% of control level), although the effect was not quite significant $(P=0.056$; Fig. $3 \mathrm{~A})$ perhaps due to a variability in the siRNA delivery within the tumor. There was no difference in mouse RLN receptor Rxfpl expression in

tumor RNA (Fig. 3A) confirming the specificity of siRXFP1. IHC with specific monoclonal anti-RXFP1 antibody revealed a significant reduction in receptor expression. There was a strong correlation of the data obtained by qRT-PCR and IHC for individual xenografts (Fig. 3B).

Treatment with siRXFP1s in this experiment did not induce classic interferon target gene $2^{\prime} 5^{\prime}$-oligoadenylate synthetase, OSA1, suggesting that the delivery of siRNA complexes did not trigger a substantial type I interferon response under these conditions (Bridge et al. 2003, Sledz et al. 2003, Pebernard \& Iggo 2004). There was no difference in the expression of OSAl gene between the control and experimental group at RNA level based on results of qRT-PCR analysis (data not shown).

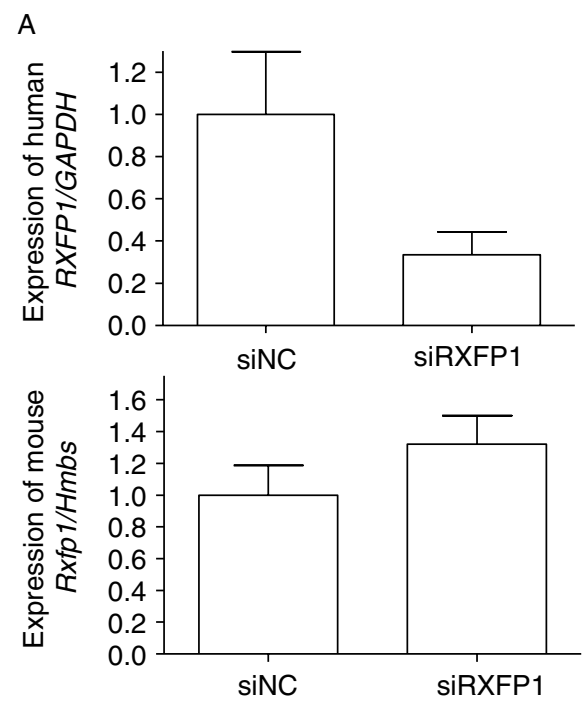

B

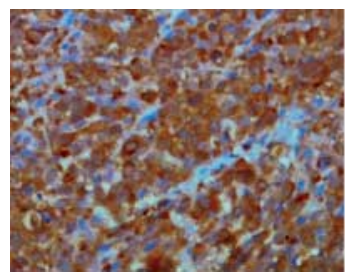

siNC

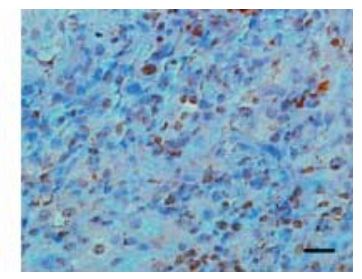

SiRXFP1
Figure 3 The siRXFP1 suppressed the expression of human $R X F P 1$ gene in LNCaP xenograft tumors. (A) Downregulation of human RXFP1 gene expression in LNCaP tumors after seven injections with siRXFP1-CNNP1 treatment $(P=0.056)$. There was no downregulation of mouse Rxfp1 gene in the host cells in xenografts. siNC-CNNP, $n=10$; siRXFP1-CNNP1, $n=7$. Error bars denote \pm s.E.M. (B) Representative sections of siRNA-CNNP-treated tumors stained with anti-RXFP1 monoclonal antibody showing a significant reduction in RXFP1 expression in siRXFP1-CNNP-treated xenografts. Magnification, $\times 400$. 


\section{Effect of siRXFP1 on in vivo growth of PC3 xenografts}

Two independent experiments were performed with PC3 xenografts and different batches of siRXFP1-0 and chitosan-siRNA preparations; similar findings were obtained in both the experiments and, thus, the results were combined (total of 20 mice in each group). The higher tumorigenicity of PC3 cells resulted in higher tumor taking rate $(89 \%, 40 / 45)$. Tumor volumes were measured during the course of the experiment (details see Materials and methods). As shown in Fig. 4A, tumor growth was significantly decreased 1 week after siRXFP1-0-CNNP injection and only a moderate increase in tumor size in the experimental group was observed until the last (6th) injection. Notably, the tumors in the siRXFP1-0-CNNP treatment group partially resumed the growth potential after the termination of siRXFP1 treatment indicating
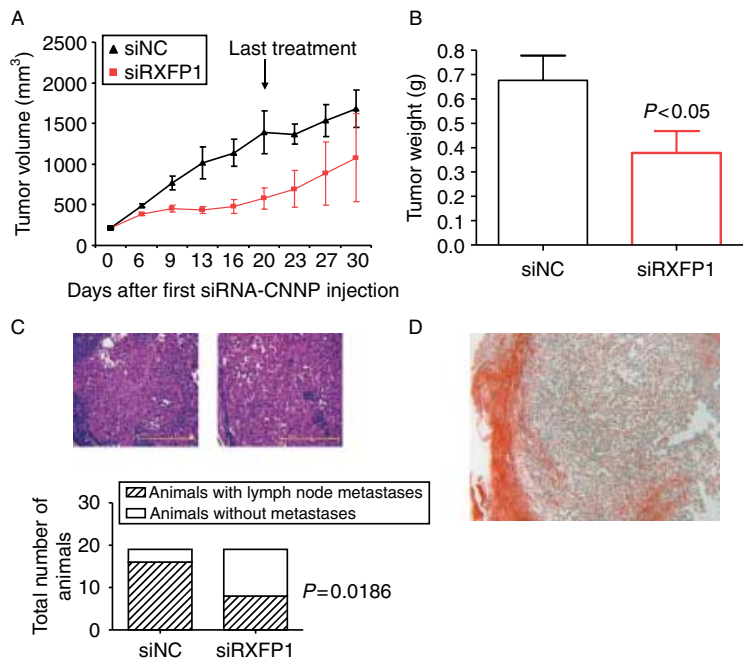

D

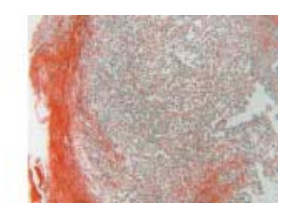

Figure 4 Suppression of in vivo tumor growth by siRXFP1-CNNP in androgen-receptor-negative PC3 xenograft tumors. (A) Intratumoral injection of siRXFP1-0-CNNP inhibited the growth of $\mathrm{PC} 3$ cell xenografts. $\mathrm{PC} 3\left(10^{7}\right.$ cells/injection site $)$ in $200 \mu$ I DMEM/F12:Matrigel B (1:1) were inoculated s.c. into the right flank of nude mice. Two weeks later, the tumor-borne mice ( $n=20$ in each group) were treated with siRXFP1-1-CNNP and control siNC-CNNP bi-weekly for 3 weeks (six injections). Tumor volumes were measured by digital caliper. The difference in the tumor volume was significant $(P<0.05)$ from days 6 to 23 . (B) Reduction in tumor weight in the siRXFP1-treated group after resection at the end of experiment $(P<0.05)$. (C) Decrease in metastasis rates in siRXFP1-CNNP-treated animals. Metastases were exclusively detected in the cervical lymph nodes shown above. The difference between the siRXFP1-CNNP and siNC-CNNP groups was statistically significant $(P=0.0186)$. Yellow bars correspond to $200 \mu \mathrm{m}$. (D) Small tumors in the siRXFP1-CNNP-treated group encapsulated in a thick collagen layer as shown with picrosirius red staining. Magnification, $\times 400$. Error bars denote \pm S.E.M. that the RXFP1 suppression effect might be transient and reversible.

On termination of the experiments, the primary tumor weight in the siNC-CNNP group was $60 \%$ higher than that in the siRXFP1-0-CNNPtreated group $(P<0.05$; Fig. 4B). The relative RXFP1/GAPDH expression within the tumor did not reveal significant differences between the groups (siNC-CNNP: $0.18 \pm 0.03$ versus siRXFP1-0-CNNP: $0.21 \pm 0.08$ relative arbitrary units), suggesting that the $R X F P 1$ expression was normalized 9 days after the termination of siRNA treatment.

One of the most consistent RLN effects on cancer cells in vitro is an increased cell migration, matrix penetration, and cell adhesion (Klonisch et al. 2007). Alternatively, the suppression of RLN or RXFP1 expression in cancer cells by siRNA downregulation caused decreased cell migration and invasiveness (Kamat et al. 2006, Feng et al. 2007). We analyzed the nude mice with PC3 xenografts for signs of metastatic tissue invasion and detected metastases exclusively in the cervical lymph nodes (Fig. 4C). The incidence of metastasis in mice injected with the siRXFP1-0-CNNP was 3.6 times lower $(15.89 \%)$ than in mice injected with control siNC-CNNP $(57.9 \%, P=0.0186)$. Furthermore, primary small tumors were more frequently observed in the siRXFP1-CNNP-treated group and were encapsulated in a thick collagen layer (Fig. 4D).

The proliferation of tumor cells in primary tumors from experimental and control siRNA-treated mice was evaluated by IHC using proliferation marker Ki67. The number of Ki67-positive cells was significantly higher in the siNC-CNNP group indicating that siRXFP1-0-CNNP treatment significantly downregulated the proliferation of PC3 cells within the xenografts (Fig. 5A). The TUNEL assay was used to analyze cell apoptosis in xenografts. Suppression of RXFP1 by siRXFP1-0-CNNP treatment significantly increased the rates of apoptosis in these prostate cancer cells (Fig. 5B). We did not find significant differences in the vascularization between the siRXFP1-0-CNNP and siNC-CNNP-treated PC3 xenografts as determined by CD34 IHC staining (Fig. 5C). Thus, the reduced PC3 tumor growth during siRXFP1-CNNP treatment was mainly the result of decreased proliferation and enhanced apoptosis of PC3 cells.

\section{Gene expression profiling of PC3 cells after downregulation of RXFP1}

To identify the potential molecular mechanisms involved in tumor cell inhibitory effects of siRNA against RLN receptor, we performed the global gene 


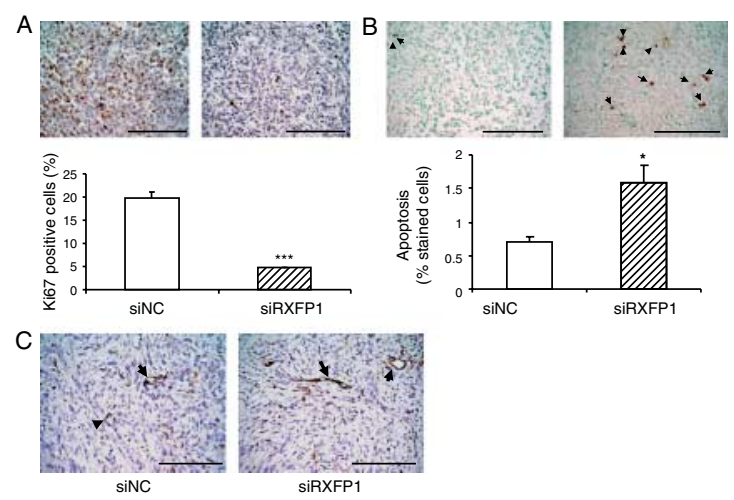

Figure 5 Effect of siRXFP1 treatment on tumor cell proliferation, apoptosis, and tumor vascularization in PC3 xenografts. (A) Suppression of tumor cell proliferation in siRXFP1-CNNP-treated animals revealed by the proliferation marker Ki67 (siNC-CNNP, $n=15$; siRXFP1-CNNP, $n=12$;

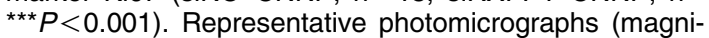
fication, $\times 400$ ) showing immunohistochemical dark brown staining for Ki67 in tumor sections of siNC-CNNP and siRXFP1-CNNP-treated mice. (B) Increased apoptosis in tumors in siRXFP1-CNNP-treated animals detected by the terminal deoxynucleotidyl transferase-mediated dUTP nick end labeling assay (siNC-CNNP, $n=8$; siRXFP1-CNNP, $n=8,{ }^{\star} P<0.05$ ). Black arrows point to the dark brown stained apoptotic cells. (C) siRXFP1-CNNP treatment did not affect vascularization in tumor tissues. Arrows indicate blood vessels. Representative photomicrographs are shown. Black bars on all images correspond to $200 \mu \mathrm{m}$. Error bars denote \pm s.E.M.

expression analysis of $\sim 22000$ RNA transcripts in PC3 cells transfected with siRXFP1-0 and siNC. This treatment did not significantly affect viability of the prostate cancer cells (Feng et al. 2007). Forty-eight hours after siRNA transfection, total RNA was isolated and qRT-PCR confirmed an $80 \%$ suppression of $R X F P 1$ gene expression in cells treated with siRXFP1 as compared with treatment with control siNC (data not shown). In the siRXFP1-treated group, 78 genes appeared downregulated and 21 genes were upregulated more than 1.3 fold (each RXFP1 profile was compared with each of the siNC profiles; Fig. 6A, complete gene list in Supplementary Table 2, see section on supplementary data given at the end of this article). The 1.3 cutoff fold was determined based on qRT-PCR results. The array data were then confirmed for a selected number of genes using qRT-PCR analysis (Fig. 6B). The potential target genes included a number of genes previously implicated in cancer progression. These included type 1 transmembrane protein, MUC1; melanoma cell adhesion molecule, MCAM (MUC18); tumor-associated antigen CO-029, TSPAN8; glucose phosphate isomerase, GPI; and angiopoietin-like 4, ANGPTL4.

\section{Discussion}

The growth and progression of prostate cancer is influenced by an array of endogenous growth factors and hormones. We have studied the tumorigenic effect of RLN, a small peptide hormone produced both in the normal prostate and in prostate cancer (Figueiredo et al. 2005, Silvertown et al. 2006, Thompson et al. 2006, Vinall et al. 2006, Feng et al. 2007, Liu et al. 2008). Previously it has been shown that the RLN expression was increased in aggressive metastatic disease (Figueiredo et al. 2005, Thompson et al. 2006, Feng et al. 2007), and that the stimulation of prostate cancer cells with RLN accelerated their invasiveness, adhesion, survival, and decreased cell apoptosis (Feng et al. 2007). Moreover, in the TRAMP mouse model, transgenic overexpression of RLN decreased survival of the males with prostate cancer (Feng et al. 2007). In this study, we used the in vivo nude xenograft mouse model to analyze the effect of RLN receptor suppression on the growth of two commonly used prostate adenocarcinoma cell lines, AR-positive LNCaP cells and AR-negative PC3 cancer cells. The effect of RXFP1 downregulation on tumor spread was also evaluated in PC3 model prone to metastasis. The targeted downregulation of human RXFP1 in prostate cancer cell xenografts by means of specific siRNA against $R X F P 1$ coated to CNNP resulted in significantly decreased tumor growth and metastasis rate in nude mice. The downregulation of RLN receptor RXFP1 in tumor cells may be a novel strategy in prostate cancer therapy.

RLN hormone produced in prostate cells signals through G-protein-coupled receptor RXFP1 (Hsu et al. 2002). The closely related insulin-like 3 peptide receptor RXFP2 also responds to higher doses of RLN by cAMP increase in cells transfected with RXFP2 (Feng et al. 2007), however, such crossreactivity was not detected in vivo (Kamat et al. 2004, Feng et al. 2006). Despite significant variations in the amino acid sequence of different mammalian RLN peptides, they all efficiently cross-activate human RXFP1 (Sherwood 2004, Bathgate et al. 2006). Recently, it was demonstrated that an analog of RLN peptide with a mutated receptor-binding domain has a moderately suppressive effect on prostate tumor when stably or transiently expressed in prostate cancer cells (Silvertown et al. 2006). With apparent limitations of an overexpression approach in mind, we decided to investigate the effect of direct RXFP1 knockdown in prostate cancer cells. The siRNA approach provided an opportunity to target RLN receptor specifically in prostate cancer cells, since the siRNA-RXFP1 
A

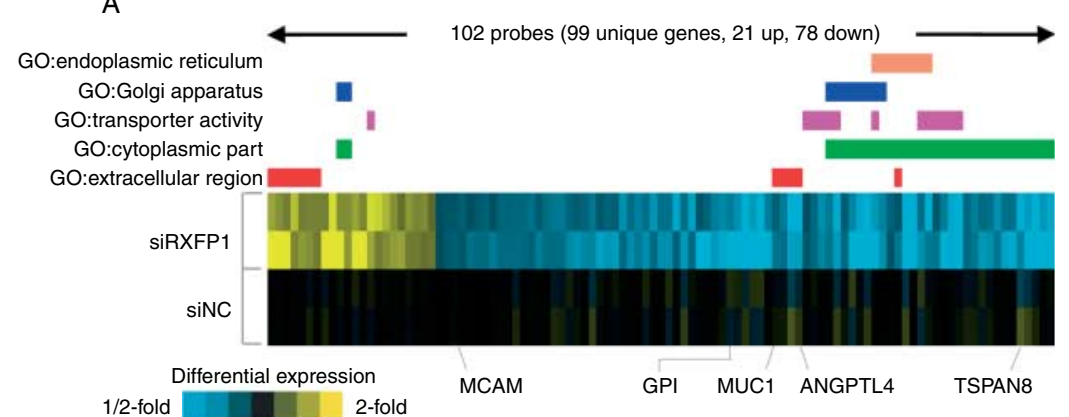

B

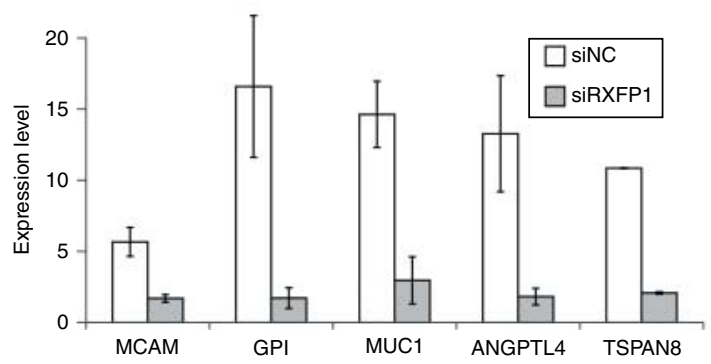

Figure 6 Global transcriptional profiling of PC3 cells treated with siRXFP1 revealed genes previously shown to promote tumorigenesis. (A) Expression data matrix ('heat map') of 99 genes differentially expressed between siRXFP1 and siNC-treated PC3 cells. Each column represents a gene and each row a sample profile. The relative level of expression of each gene in each treatment group is represented using a yellow-blue color scale (black: no change; blue: low expression, bright yellow $>2$-fold from control). Color bars above the matrix denote the corresponding annotation of genes using selected Gene Ontology (GO) terms. (B) RT-PCR validation of selected gene targets in PC3 cells $48 \mathrm{~h}$ after siRNA transfection. Expression differences between siRXFP1 and siNC were significant with $P<0.05$ for each gene. Error bars denote \pm s.E.M. Expression differences between siRXFP1 and siNC were significant with $P<0.05$ for each gene.

construct utilized in this study had little homology with the mouse RXFP1 sequence. Indeed, the data showed specific downregulation of RXFP1 in human prostate cancer cells but had no effect on the expression of mouse RLN receptor. For siRNA delivery, we have used the recently developed approach of biodegradable CNNP (Howard et al. 2006, Katas \& Alpar 2006, Dass \& Choong 2008, Katas et al. 2008, de Martimprey et al. 2008). In these experiments we have directly injected nanoparticales into tumor tissues. Such an approach will be difficult to apply to a clinical setting; however, it allowed us to achieve maximum downregulation of $R X F P 1$ target gene and provided a proof of principle that RXFP1 targeting may be clinically useful in prostate cancer. We have found that a single injection of siRXFP1 into established PC3 human prostate cancer xenografts in nude males lead to a 35 and $60 \%$ reduction in RXFPl mRNA 24 and $48 \mathrm{~h}$ after siRNA injection respectively. Bi-weekly injections of siRXFP1-CNNP into the tumors significantly slowed the growth of prostate adenocarcinoma xenografts. Already after two injections, tumors ceased to increase in volume and either shrank as in LNCaP cells or only grew slightly (PC3) by the end of third week. Previous observations had shown the in vitro suppressive effects of impaired RLN signaling on migration, invasiveness, adhesion, and other characteristics affecting the dissemination of cancer cells (Klonisch et al. 2007). In this study, we show a significant reduction in metastasis rates in tumors treated with RXFP1-CNNP complexes in metastasis-prone $\mathrm{PC} 3$ cells in vivo. The siRXFP-CNNP group displayed a significantly reduced $(42.3 \%)$ rate of cervical lymph node metastases as compared with the siNC-CNNP group, clearly implicating RLN-RXFP1 signaling pathways as important players in tumor cell metastasis in mice.

The broad range effects of RLN signaling on cancer cell proliferation, survival, apoptosis, ECM remodeling, and tumor angiogenesis are well-established (Silvertown et al. 2003, Klonisch et al. 2007). In the PC3 xenograft mouse model, suppression of RLN signaling leads to increased apoptosis and decreased cellular proliferation. The smaller tumors observed with siRXFP1-CNNP treatment were encapsulated in a thick picrosirius-positive layer indicating higher collagen deposition. We did not detect increased tumor vascularization in this model as has been reported previously in experiments that used overexpression of a mutant RLN peptide with antagonistic properties to suppress RLN signaling (Silvertown et al. 2007). 
One possible explanation is that in our model RLN signaling was suppressed only in tumor cells but not in the host tissues as a result of the siRNA effectively and specifically depleting human $R X F P 1$, with little or no effect on mouse Rxfpl transcripts. On the other hand, the peptide antagonist used in the previous study (Silvertown et al. 2007) might have suppressed RLN responses in both human tumor and mouse host cells and thus may have caused lower tumor vascularization and angiogenesis in that model. This may suggest the intriguing possibility of an active role of RLN signaling in modulating host cell-tumor cell interactions resulting in specific responses, including tumor progression and tumor angiogenesis.

It was shown previously that the short hairpin RNAs (shRNAs) or siRNAs may induce an interferon response in transfected cells (Bridge et al. 2003, Sledz et al. 2003, Pebernard \& Iggo 2004). To exclude such nonspecific effects, the expression level of classical interferon target gene OSAl was measured in cells transfected with siRNA and in tumors treated with siNC/siRXFP1-CNNP complexes. No differences were detected in the experiments with two cell lines. In addition, the microarray analyses performed on PC3 cells did not reveal significant upregulation in the expression of known target genes of the interferon pathway. The two siRXFP1 siRNAs did not affect the expression of host mouse Rxfpl gene in xenografts, indicating the absence of off-target effects.

The histological analysis of LNCaP tumor sections revealed that the majority of tumors treated with siRXFP1-CNNP presented with extensive central necrosis suggesting the demise of tumor cells as a result of siRXFP1 nanoparticle uptake in close vicinity to the injection sites. Control siNC-CNNP-treated tumors were mainly composed of densely packed epithelial tumor cells indicating that neither the CNNP nor the siNC-CNNP nanoparticles were toxic to the tumor cells. In conclusion, siRXFP1-CNNP may be more effective in tumors derived from LNCaP cells causing more extensive necrosis. By contrast, PC3 cells are possibly more resistant to the actions of siRXFP1-CNNP and this could explain the most subtle tumor tissue response observed in these prostate cancer cells. One of the major differences between two cell lines, $\mathrm{PC} 3$ and $\mathrm{LNCaP}$, is the presence of active AR signaling in LNCaP cells. It was shown that RLN was acting through RXFP1 to stimulate androgenindependent growth of LNCaP cells by a mechanism that involved the activation of the AR signaling pathway. The specific mechanisms by which RLN facilitates tumor growth may be different in different cancer cells. Previously it was shown that PI3K/Akt and the components of the Wnt pathway could be responsible for RLN-mediated activation of the AR pathway in androgen-responsive $\mathrm{LNCaP}$ prostate cancer cells (Liu et al. 2008). We have shown that in AR-negative PC3 cells the inactivation of the RLN receptor also caused a decrease in Ser308 Akt phosphorylation (Agoulnik AA, Feng S, unpublished data, 2009). It is likely, therefore, that the RLN effects on prostate cancer cells are mediated through both AR-dependent (Liu et al. 2008) and ARindependent pathways.

Analysis of differentially expressed genes following siRXFP1 treatment revealed several potential targets of RLN signaling in PC3 prostate cancer cells. Examples of these novel RLN-modulated genes uncovered by microarrays and confirmed by qRT-PCR in PC3 cells included several downregulated genes with well-known function in oncogenesis. This includes MUC1, a type 1 transmembrane protein, overexpressed in a nonpolarized manner in many tumor cells (Gendler 2001). MUC1 is generally thought of having anti-adhesive effects on cell-cell and cell-substrate interactions resulting in altered cell signaling, tumor growth, and metastasis (Wesseling et al. 1996, Singh et al. 2006). In prostate cancer, enhanced expression of MUC1 correlates with increased Gleason score (Burke et al. 2006). MUC1 activates PI3K/Akt signaling (Raina et al. 2004), which correlates with our observation that suppression of RXFP1 causes inhibition of Akt phosphorylation. Another interesting target is MUC18, a cell adhesion molecule of the immunoglobulin gene superfamily (Johnson et al. 1993). MUC18 has also been shown to be overexpressed in human prostate cancer (Wu et al. 2001a,b). MUC18 expression correlates with the progression of human prostate cancer and is an important mediator for the metastatic potential of human and mouse prostate cancer cells (Wu et al. 2001 $a, b, 2004,2005$ ). The TSPAN8 (tumor-associated antigen CO-029), a member of the transmembrane 4 (tetraspanin) superfamily, is associated with a poor cancer prognosis (Claas et al. 1998, Kanetaka et al. 2001, Gesierich et al. 2005, Kuhn et al. 2007). Tumor cell-derived ANGPTL4 disrupts vascular endothelial cell-cell junctions to facilitate the metastasis of tumor cells (Padua et al. 2008). GPI is thought to facilitate tumor cell invasion and metastasis (Funasaka et al. 2002). The downregulation of all these targets observed following silencing of RLN signaling further supports the potential of siRXFP1-CNNP for prostate cancer therapy in men.

In summary, our data demonstrated that downregulation of RXFP1 RLN receptor by siRNA on 
CNNP effectively suppressed tumor growth in vivo through increased apoptosis and decreased proliferation of PC3 cancer cells. Furthermore, the suppression of RLN signaling significantly reduced metastasis rates. We have identified several RLN responsive genes in prostate cancer cells with defined effects on tumor progression implicating RLN and its receptor RXFP1 as potential therapeutic targets for prostate cancer treatment.

\section{Supplementary data}

This is linked to the online version of the paper at http://dx. doi.org/10.1677/ERC-10-0073.

\section{Declaration of interest}

The authors declare that there is no conflict of interest that could be perceived as prejudicing the impartiality of the research reported.

\section{Funding}

This study was supported by the National Cancer Institute grants R21CA118362 to A I Agoulnik, P50CA058204 to M M Ittmann, NSERC342187 Natural Sciences and Engineering Research Council of Canada to T Klonisch, Program Project Development Grant from the Ovarian Cancer Research Fund, Inc. to A K Sood, and the China Scholarship Council to Z Li.

\section{Acknowledgements}

We thank University of Texas Health Science Center at Houston Microarray Core Laboratory for the help with microarray analysis, Baylor College of Medicine Tissue and Cell Culture Core Laboratory, and Dr Carolyn Smith for providing prostate cancer cells. The authors thank Sukhjinder Kaur for technical support.

\section{References}

Agoulnik AI 2007 Relaxin and related peptides in male reproduction. Advances in Experimental Medicine and Biology 612 49-64.

Bathgate RA, Ivell R, Sanborn BM, Sherwood OD \& Summers RJ 2006 International Union of Pharmacology LVII: recommendations for the nomenclature of receptors for relaxin family peptides. Pharmacological Reviews $\mathbf{5 8}$ 7-31. (doi:10.1124/pr.58.1.9)

Bridge AJ, Pebernard S, Ducraux A, Nicoulaz AL \& Iggo R 2003 Induction of an interferon response by RNAi vectors in mammalian cells. Nature Genetics 34 263-264. (doi:10.1038/ng1173)

Burke PA, Gregg JP, Bakhtiar B, Beckett LA, Denardo GL, Albrecht H, De Vere White RW \& De Nardo SJ 2006
Characterization of MUC1 glycoprotein on prostate cancer for selection of targeting molecules. International Journal of Oncology 29 49-55.

Claas C, Seiter S, Claas A, Savelyeva L, Schwab M \& Zoller M 1998 Association between the rat homologue of CO-029, a metastasis-associated tetraspanin molecule and consumption coagulopathy. Journal of Cell Biology 141 267-280. (doi:10.1083/jcb.141.1.267)

Dass CR \& Choong PF 2008 The use of chitosan formulations in cancer therapy. Journal of Microencapsulation 25 275-279. (doi:10.1080/02652040801970461)

Feng S, Bogatcheva NV, Kamat AA \& Agoulnik AI 2005 Genetic targeting of relaxin and insl3 signaling in mice. Annals of the New York Academy of Sciences 1041 82-90. (doi:10.1196/annals.1282.012)

Feng S, Bogatcheva NV, Kamat AA, Truong A \& Agoulnik AI 2006 Endocrine effects of relaxin overexpression in mice. Endocrinology 147 407-414. (doi:10.1210/en. 2005-0626)

Feng S, Agoulnik IU, Bogatcheva NV, Kamat AA, Kwabi-Addo B, Li R, Ayala G, Ittmann MM \& Agoulnik AI 2007 Relaxin promotes prostate cancer progression. Clinical Cancer Research 13 1695-1702. (doi:10.1158/1078-0432.CCR-06-2492)

Figueiredo KA, Palmer JB, Mui AL, Nelson CC \& Cox ME 2005 Demonstration of upregulated $\mathrm{H} 2$ relaxin mRNA expression during neuroendocrine differentiation of LNCaP prostate cancer cells and production of biologically active mammalian recombinant 6 histidine-tagged $\mathrm{H} 2$ relaxin. Annals of the New York Academy of Sciences 1041 320-327. (doi:10.1196/annals.1282.051)

Funasaka T, Haga A, Raz A \& Nagase H 2002 Tumor autocrine motility factor induces hyperpermeability of endothelial and mesothelial cells leading to accumulation of ascites fluid. Biochemical and Biophysical Research Communications 293 192-200. (doi:10.1016/S0006291X(02)00202-4)

Gendler SJ 2001 MUC1, the renaissance molecule. Journal of Mammary Gland Biology and Neoplasia 6 339-353. (doi:10.1023/A:1011379725811)

Gesierich S, Paret C, Hildebrand D, Weitz J, Zgraggen K, Schmitz-Winnenthal FH, Horejsi V, Yoshie O, Herlyn D, Ashman LK et al. 2005 Colocalization of the tetraspanins, CO-029 and CD151, with integrins in human pancreatic adenocarcinoma: impact on cell motility. Clinical Cancer Research 11 2840-2852. (doi:10.1158/1078-0432.CCR04-1935)

Giordano TJ, Thomas DG, Kuick R, Lizyness M, Misek DE, Smith AL, Sanders D, Aljundi RT, Gauger PG, Thompson NW et al. 2003 Distinct transcriptional profiles of adrenocortical tumors uncovered by DNA microarray analysis. American Journal of Pathology 162 521-531.

Hansell DJ, Bryant-Greenwood GD \& Greenwood FC 1991 Expression of the human relaxin $\mathrm{H} 1$ gene in the decidua, trophoblast, and prostate. Journal of Clinical Endocrinology and Metabolism 72 899-904. (doi:10.1210/ jcem-72-4-899) 
Howard KA, Rahbek UL, Liu X, Damgaard CK, Glud SZ, Andersen MO, Hovgaard MB, Schmitz A, Nyengaard JR, Besenbacher F et al. 2006 RNA interference in vitro and in vivo using a novel chitosan/siRNA nanoparticle system. Molecular Therapy 14 476-484. (doi:10.1016/j.ymthe. 2006.04.010)

Hsu SY, Nakabayashi K, Nishi S, Kumagai J, Kudo M, Sherwood OD \& Hsueh AJ 2002 Activation of orphan receptors by the hormone relaxin. Science 295 671-674. (doi:10.1126/science.1065654)

Johnson JP, Rothbacher U \& Sers C 1993 The progression associated antigen MUC18: a unique member of the immunoglobulin supergene family. Melanoma Research 3 337-340. (doi:10.1097/00008390-199310000-00006)

Kamat AA, Feng S, Bogatcheva NV, Truong A, Bishop CE \& Agoulnik AI 2004 Genetic targeting of relaxin and insulin-like factor 3 receptors in mice. Endocrinology 145 4712-4720. (doi:10.1210/en.2004-0515)

Kamat AA, Feng S, Agoulnik IU, Kheradmand F, Bogatcheva NV, Coffey D, Sood AK \& Agoulnik AI 2006 The role of relaxin in endometrial cancer. Cancer Biology \& Therapy 5 71-77. (doi:10.4161/cbt.5.1.2289)

Kanetaka K, Sakamoto M, Yamamoto Y, Yamasaki S, Lanza F, Kanematsu T \& Hirohashi S 2001 Overexpression of tetraspanin CO-029 in hepatocellular carcinoma. Journal of Hepatology 35 637-642. (doi:10. 1016/S0168-8278(01)00183-0)

Katas H \& Alpar HO 2006 Development and characterisation of chitosan nanoparticles for siRNA delivery. Journal of Controlled Release 115 216-225. (doi:10.1016/j.jconrel. 2006.07.021)

Katas H, Chen S, Osamuyimen AA, Cevher E \& Alpar HO 2008 Effect of preparative variables on small interfering RNA loaded poly(D,L-lactide-co-glycolide)-chitosan submicron particles prepared by emulsification diffusion method. Journal of Microencapsulation 25 541-548. (doi:10.1080/02652040802075567)

Klonisch T, Froehlich C, Tetens F, Fischer B \& HombachKlonisch S 2001 Molecular remodeling of members of the relaxin family during primate evolution. Molecular Biology and Evolution 18 393-403.

Klonisch T, Bialek J, Radestock Y, Hoang-Vu C \& HombachKlonisch S 2007 Relaxin-like ligand-receptor systems are autocrine/paracrine effectors in tumor cells and modulate cancer progression and tissue invasiveness. Advances in Experimental Medicine and Biology 612 104-118.

Kuhn S, Koch M, Nubel T, Ladwein M, Antolovic D, Klingbeil P, Hildebrand D, Moldenhauer G, Langbein L, Franke WW et al. 2007 A complex of EpCAM, claudin-7, CD44 variant isoforms, and tetraspanins promotes colorectal cancer progression. Molecular Cancer Research 5 553-567. (doi:10.1158/1541-7786.MCR-06-0384)

Liu S, Vinall RL, Tepper C, Shi XB, Xue LR, Ma AH, Wang LY, Fitzgerald LD, Wu Z, Gandour-Edwards R et al. 2008 Inappropriate activation of androgen receptor by relaxin via $\beta$-catenin pathway. Oncogene 27 499-505. (doi:10.1038/sj.onc.1210671)
Lu C, Han HD, Mangala LS, Ali-Fehmi R, Newton CS, Ozbun L, Armaiz-Pena GN, Hu W, Stone RL, Munkarah A et al. 2010 Regulation of tumor angiogenesis by EZH2. Cancer Cell 18 185-197. (doi:10.1016/j.ccr.2010.06.016)

de Martimprey H, Bertrand JR, Fusco A, Santoro M, Couvreur P, Vauthier C \& Malvy C 2008 siRNA nanoformulation against the ret/PTC1 junction oncogene is efficient in an in vivo model of papillary thyroid carcinoma. Nucleic Acids Research 36 e2. (doi:10.1093/ nar/gkm1094)

Padua D, Zhang XH, Wang Q, Nadal C, Gerald WL, Gomis RR \& Massague J 2008 TGF $\beta$ primes breast tumors for lung metastasis seeding through angiopoietin-like 4. Cell 133 66-77. (doi:10.1016/j.cell.2008.01.046)

Pebernard S \& Iggo RD 2004 Determinants of interferonstimulated gene induction by RNAi vectors. Differentiation 72 103-111. (doi:10.1111/j.1432-0436.2004.07202001.x)

Raina D, Kharbanda S \& Kufe D 2004 The MUC1 oncoprotein activates the anti-apoptotic phosphoinositide 3-kinase/Akt and Bcl-xL pathways in rat 3Y1 fibroblasts. Journal of Biological Chemistry 279 20607-20612. (doi:10.1074/jbc.M310538200)

Saldanha AJ 2004 Java Treeview - extensible visualization of microarray data. Bioinformatics 20 3246-3248. (doi:10.1093/bioinformatics/bth349)

Sherwood OD 2004 Relaxin's physiological roles and other diverse actions. Endocrine Reviews 25 205-234. (doi:10.1210/er.2003-0013)

Silvertown JD, Summerlee AJ \& Klonisch T 2003 Relaxinlike peptides in cancer. International Journal of Cancer 107 513-519. (doi:10.1002/ijc.11424)

Silvertown JD, Ng J, Sato T, Summerlee AJ \& Medin JA $2006 \mathrm{H} 2$ relaxin overexpression increases in vivo prostate xenograft tumor growth and angiogenesis. International Journal of Cancer 118 62-73. (doi:10. 1002/ijc.21288)

Silvertown JD, Symes JC, Neschadim A, Nonaka T, Kao JC, Summerlee AJ \& Medin JA 2007 Analog of H2 relaxin exhibits antagonistic properties and impairs prostate tumor growth. FASEB Journal 21 754-765. (doi:10.1096/ fj.06-6847com)

Singh AP, Chauhan SC, Bafna S, Johansson SL, Smith LM, Moniaux N, Lin MF \& Batra SK 2006 Aberrant expression of transmembrane mucins, MUC1 and MUC4, in human prostate carcinomas. Prostate 66 421-429. (doi:10.1002/pros.20372)

Sledz CA, Holko M, de Veer MJ, Silverman RH \& Williams BR 2003 Activation of the interferon system by shortinterfering RNAs. Nature Cell Biology 5 834-839. (doi:10.1038/ncb1038)

Thompson VC, Morris TG, Cochrane DR, Cavanagh J, Wafa LA, Hamilton T, Wang S, Fazli L, Gleave ME \& Nelson CC 2006 Relaxin becomes upregulated during prostate cancer progression to androgen independence and is negatively regulated by androgens. Prostate 66 1698-1709. (doi:10.1002/pros.20423) 
Vinall RL, Tepper CG, Shi XB, Xue LA, Gandour-Edwards R \& de Vere White RW 2006 The R273H p53 mutation can facilitate the androgen-independent growth of $\mathrm{LNCaP}$ by a mechanism that involves $\mathrm{H} 2$ relaxin and its cognate receptor LGR7. Oncogene 25 2082-2093. (doi:10.1038/ sj.onc.1209246)

Wesseling J, van der Valk SW \& Hilkens J 1996 A mechanism for inhibition of E-cadherin-mediated cellcell adhesion by the membrane-associated mucin episialin/MUC1. Molecular Biology of the Cell 7 565-577.

Wu GJ, Varma VA, Wu MW, Wang SW, Qu P, Yang H, Petros JA, Lim SD \& Amin MB 2001 $a$ Expression of a human cell adhesion molecule, MUC18, in prostate cancer cell lines and tissues. Prostate 48 305-315. (doi:10.1002/pros.1111)
Wu GJ, Wu MW, Wang SW, Liu Z, Qu P, Peng Q, Yang H, Varma VA, Sun QC, Petros JA et al. 2001 Isolation and characterization of the major form of human MUC18 cDNA gene and correlation of MUC18 over-expression in prostate cancer cell lines and tissues with malignant progression.

Gene 279 17-31. (doi:10.1016/S0378-1119(01)00736-3)

Wu GJ, Peng Q, Fu P, Wang SW, Chiang CF, Dillehay DL \& Wu MW 2004 Ectopical expression of human MUC18 increases metastasis of human prostate cancer cells. Gene 327 201-213. (doi:10.1016/j.gene.2003.11.018)

Wu GJ, Fu P, Chiang CF, Huss WJ, Greenberg NM \& Wu MW 2005 Increased expression of MUC18 correlates with the metastatic progression of mouse prostate adenocarcinoma in the TRAMP model. Journal of Urology 173 1778-1783. (doi:10.1097/01.ju. $0000154643.30048 .2 \mathrm{c})$ 